\title{
TT-TENSORS AND CONFORMALLY FLAT STRUCTURES ON 3-MANIFOLDS
}

\author{
R. BEIG \\ Institut für Theoretische Physik \\ Universität Wien, Austria \\ Boltzmanngasse 5, A-1090 Wien, Austria \\ E-mail: beig@pap.univie.ac.at
}

\begin{abstract}
We study TT-tensors on conformally flat 3-manifolds $(M, g)$. The Cotton-York tensor linearized at $g$ maps every symmetric tracefree tensor into one which is TT. The question as to whether this is the general solution to the TT-condition is viewed as a cohomological problem within an elliptic complex first found by Gasqui and Goldschmidt and reviewed in the present paper. The question is answered affirmatively when $M$ is simply connected and has vanishing 2nd de Rham cohomology.
\end{abstract}

1. Introduction. In the context of the initial-value problem for the Einstein equations (see [5]) one is often interested in the following problem. Let $(M, g)$ be a connected, smooth, compact, 3-dimensional, orientable manifold and let $t_{a b}$ be an element of $S_{0}^{2}(M, g)$, that is to say a 2-covariant, symmetric tensor field which is tracefree with respect to $g_{a b}$, i.e. $t_{a b}=t_{(a b)}$ and $t_{a b} g^{a b}=0$, where $g^{a b}$ is the inverse of $g_{a b}$. We want to solve the equation

$$
(\delta t)_{a}:=2 g^{b c} D_{c} t_{a b}=0,
$$

where $D_{a}$ is the Levi-Civita connection associated with $g_{a b}$. Elements of $S_{0}^{2}(M, g)$ satisfying (1.1) are also called TT-tensors. The equation (1.1) is an underdetermined elliptic system. This means that the principal symbol of $\delta$, namely the linear map

$$
\bar{\delta}(k ; x): \tau \in S_{0}^{2}\left(\mathbf{R}^{3}, g_{x}\right) \rightarrow \omega \in \Lambda^{1}\left(\mathbf{R}^{3}\right), \quad k \in \Lambda^{1}\left(\mathbf{R}^{3}\right), \quad k \neq 0,
$$

defined by

$$
\omega_{a}=g^{b c}(x) k_{c} \tau_{a b}
$$

1991 Mathematics Subject Classification: 53A30, 83C05.

Supported by Fonds zur Förderung der wissenschaftlichen Forschung, Project P9376-MAT.

The paper is in final form and no version of it will be published elsewhere. 
is surjective. ${ }^{1}$ There is a general method (see the Appendix of [2]) to solve such a system, as follows: Define the operator $L$

$$
L: \Lambda^{1}(M) \rightarrow S_{0}^{2}(M, g)
$$

by

$$
(L W)_{a b}=D_{a} W_{b}+D_{b} W_{a}-\frac{2}{3} g_{a b} D^{c} W_{c} .
$$

Clearly $-L=\delta^{*}$, i.e. $L$ is minus the formal adjoint of $\delta$ under the inner product given by the Riemannian volume element of $g$. The kernel of $L$ is the finite-dimensional space of covector fields $W_{a}$, so that $W^{a}=g^{a b} W_{b}$ is a conformal Killing vector field on $(M, g)$. Furthermore there is the decomposition

$$
S_{0}^{2}(M, g)=L\left(\Lambda^{1}(M)\right) \oplus \operatorname{ker} \delta .
$$

Starting with an element $Q_{a b} \in S_{0}^{2}(M, g)$, its component $t_{a b}$ in $\operatorname{ker} \delta$ can formally be written as

$$
t=\left[\mathbf{1}-L(\delta \circ L)^{-1} \delta\right] Q .
$$

Since $\operatorname{ker}(\delta \circ L)=\operatorname{ker} L$ and $\delta Q$ is orthogonal to ker $L$, the right-hand side of Equ. (1.5) is well defined. The relations given by (1.3) and (1.4) furnish what is called the York decomposition (after [20], see also [6]) in the G.R. literature. This decomposition is closely related to the study of the action of conformal diffeomorphisms on the space of Riemannian metrics on $M[9]$. In the present work we seek a refinement of this decomposition in a sense which is best explained by the example of the de Rham-Hodge theory. Consider, thus, instead of (1.1), the equation

$$
\operatorname{div} \omega=D^{a} \omega_{a}=0 .
$$

Again, this is an underdetermined elliptic system, and we have the orthogonal decomposition

$$
\Lambda^{1}(M)=\operatorname{grad}\left(C^{\infty}(M)\right) \oplus \operatorname{ker} \operatorname{div},
$$

where grad is minus the formal adjoint of div, namely the differential acting on functions. Sometimes the relation (1.7) is called Helmholtz decomposition in the physics literature. The splitting given by (1.7) can be refined by noticing that there is a large class of explicit solutions to (1.6) namely all elements $\omega \in \Lambda^{1}(M)$ of the form $\omega=$ rot $\mu$, where rot: $\Lambda^{1}(M) \rightarrow \Lambda^{1}(M)$ is defined by

$$
\omega_{a}=\varepsilon_{a}{ }^{b c} D_{b} \mu_{c} .
$$

Every element in grad $\left(C^{\infty}(M)\right)$, in turn, is in the kernel of rot. Then consider the sequence of spaces and linear maps

$$
0 \rightarrow C^{\infty}(M) \stackrel{\text { grad }}{\longrightarrow} \Lambda^{1}(M) \stackrel{\text { rot }}{\longrightarrow} \Lambda^{1}(M) \stackrel{\text { div }}{\longrightarrow} C^{\infty}(M) \rightarrow 0 .
$$

\footnotetext{
${ }^{1}$ That this is the case follows by setting

$$
\tau_{a b}=\frac{2}{k^{2}} k_{(a} \omega_{b)}-\frac{1}{2 k^{2}} g_{a b}(k, \omega)-\frac{1}{2 k^{4}} k_{a} k_{b}(k, \omega),
$$
}

where $k^{2}:=g^{a b}(x) k_{a} k_{b}$ and $(k, \omega):=g^{a b}(x) \omega_{a} k_{b}$. 
This is a complex, i.e. every element in each of these spaces which is in the image of the map to the left, is also in the kernel of the map to the right. It is also an elliptic complex, i.e. the corresponding complex of symbols is exact: every element in the kernel of a symbol map to its right is in the image of the symbol map to its left. For grad and div this just amounts to the statement that div is underdetermined elliptic, and, equivalently, that grad is overdetermined elliptic (the associated symbol map is injective). Now define the Hodge Laplacian $\Delta_{H}$

$$
\Delta_{H}: \Lambda^{1}(M) \rightarrow \Lambda^{1}(M)
$$

by

$$
\Delta_{H}=(\operatorname{rot})^{2}-\operatorname{grad} \operatorname{div} .
$$

This has the following properties: It is formally self-adjoint and elliptic (i.e. its symbol is injective and surjective). Thus (see Warner [19]) ker $\Delta_{H}$ is finite-dimensional and

$$
\begin{aligned}
\Lambda^{1}(M) & =\Delta_{H}\left(\Lambda^{1}(M)\right) \oplus \operatorname{ker} \Delta_{H} \\
& =\operatorname{grad}\left(C^{\infty}(M)\right) \oplus \operatorname{rot}\left(\Lambda^{1}(M)\right) \oplus \operatorname{ker} \Delta_{H} \\
& =\operatorname{grad}\left(C^{\infty}(M)\right) \oplus \operatorname{ker} \operatorname{div} .
\end{aligned}
$$

Thus

$$
\operatorname{ker} \operatorname{div}=\operatorname{ker} \Delta_{H} \oplus \operatorname{rot}\left(\Lambda^{1}(M)\right),
$$

and this is the sought-for refinement of (1.7). The relation (1.12) also shows that the de Rham cohomology group $H^{2}=\operatorname{ker} \operatorname{div} / \operatorname{rot}\left(\Lambda^{1}(M)\right)$ is isomorphic to ker $\Delta_{H}$. In other words: the possible failure of the expression (1.8) to furnish the general solution to Equ. (1.6) is measured by the second Betti number of $M$, in particular is a topological invariant of $M$. At the same time, using the formal self-adjointness of rot and the fact that $\Delta_{H}$ and rot commute, it follows from the second line of (1.11) that

$$
\text { ker rot }=\operatorname{grad}\left(C^{\infty}(M)\right) \oplus \operatorname{ker} \Delta_{H} .
$$

Thus $H^{1}=$ ker rot/grad $\left(C^{\infty}(M)\right)$ is also isomorphic to ker $\Delta_{H}$, which is an expression of Poincaré duality in the situation at hand. Note that

$$
\operatorname{ker} \Delta_{H}=\operatorname{ker} \operatorname{div} \cap \text { ker rot. }
$$

We now ask whether a similar scenario exists for Equ. (1.1). This, indeed, turns out to be the case provided $(M, g)$ is (locally) conformally flat. The associated elliptic complex has been found by Gasqui and Goldschmidt [10] in a study of infinitesimal deformations of conformally flat structures for a general manifold of dimension $n \geq 3$. Their work starts from the left end of the complex, i.e. the conformal Killing equation $L W=0$. (Their method is to apply the Spencer-Kodaira-Quillen-Goldschmidt (see e.g. [18]) theory of overdetermined systems to $L W=0$.) In a similar vein Calabi [3] and Bérard-Bergery et al. [1] had previously considered the integrability theory of the Killing equation on $(M, g)$ when $(M, g)$ is a space of constant curvature.

Acknowledgements. At the time of the lecture given at the Banach centre I was unaware of the Gasqui-Goldschmidt work. I am indebted to Professor J.-P. Bourguignon for pointing out its existence and for helpful discussions. Furthermore I am grateful to Professor D. Burghelea for teaching me the notion of an elliptic complex in the early 
stages of this work and to Professor J. Lafontaine for important information regarding the premoduli space of conformally flat structures in the case of space forms. I also thank Professor L. Andersson for telling me of Ref. [3] and Professor S. Deser for comments on the manuscript.

In the next paragraph of this paper we describe the conformal elliptic complex which plays the same role for TT-tensors as the role played by the de Rham-Hodge complex for Equ. (1.6). Here the operator rendering explicit solutions (to the TT-condition) is $H$, the Cotton-York tensor linearized at the conformal metric $g$, viewed as a map sending tracefree symmetric tensors into themselves. When $M$ is compact, the obstruction to tensors in the image of this map to furnish the general solution of (1.1) is, by an analogue of Poincaré duality, the same as the obstruction to Killing forms to be the general elements of the null space of $H$. This, in turn, has a nice geometric interpretation: namely it is the premoduli space at $g$ of the space of conformally flat deformations of $g$. For the general, non-compact, case, but when $M$ is assumed to be simply connected, Gasqui and Goldschmidt [10] have shown that the latter cohomology, namely ker $H / L\left(\Lambda^{1}(M)\right)$, is zero. In $\S 3$ of the present paper we prove our main result. It states that, when $M$ is simply connected and its second de Rham cohomology is zero, the cohomology $\operatorname{ker} \delta / H\left(S_{0}^{2}(M, g)\right)$ vanishes. In $\S 4$ we compute this space when $(M, g)$ is a compact space-form. In the elliptic case the obstruction is found to be zero, i.e. $g$ is infinitesimally rigid as a conformally flat structure. In the flat case, where $M$ is necessarily a torus, we find the obstruction space is five-dimensional: this corresponds to flat deformations modulo constant rescalings of $g$. In the hyperbolic case the deformation space is given by the space of tracefree Codazzi tensors on $(M, g)$. This result has already been obtained by Lafontaine [14] for manifolds of general dimension $\geq 3$.

2. The conformal elliptic complex. Let now $(M, g)$ be conformally flat, with $M$ not necessarily compact. Recall that this means that each point of $M$ has a coordinate neighbourhood $x^{a}$ in which

$$
g_{a b}=\omega^{2} \delta_{a b}, \quad \omega>0,
$$

where $\delta_{a b}$ is the flat Euclidean metric. It is well known [16] that, in dimension 3, this is equivalent to the vanishing of the Cotton-York tensor $\mathcal{H}_{a b}$ defined by

$$
\mathcal{H}_{a b}=2 \varepsilon_{c d(a} D^{c} \mathcal{R}^{d}{ }_{b)}=: \operatorname{rot}_{2} \mathcal{R}_{a b}
$$

where $\mathcal{R}_{a b}$ is the Ricci curvature of $g_{a b}$. Note the following properties of $\mathcal{H}_{a b}$.

(i) $g^{a b} \mathcal{H}_{a b}=0$

(ii) $D^{a} \mathcal{H}_{a b}=0$

(iii) $\mathcal{H}_{a b}\left[\omega^{2} g\right]=\omega^{-1} \mathcal{H}_{a b}[g], \omega>0$.

Geometrically these properties arise as follows. Let $U \subset M$ be a coordinate neighbourhood and consider the Chern-Simons action

$$
S[g]=\int_{U} \varepsilon^{a b c}\left(\Gamma_{d}{ }^{e}{ }_{a} \mathcal{R}_{b c}{ }^{d}{ }_{e}-\frac{2}{3} \Gamma_{d}{ }_{a}^{e} \Gamma_{e}{ }_{b}^{f} \Gamma_{f}{ }^{d}{ }_{c}\right) \sqrt{g} d^{3} x,
$$

where $\Gamma_{b}{ }^{a}{ }_{c}$ are the Christoffel symbols in the local chart $x^{a}$. The functional $S[g]$ has the (non-obvious) properties of being invariant a) under conformal rescalings of $g$ and 
b) invariant under infinitesimal coordinate changes, provided these changes suitably approach the identity on $\partial U$. (See Chern [4] and Deser et al. [7].) Note, finally, that the Euler-Lagrange expression of $S[g]$ is nothing but $-3 \mathcal{H}^{a b}$. Then (i,iii) are implied by a) and (ii) is implied by b).

Consider, next, the linearization of $\mathcal{H}_{a b}$ at a conformally flat metric $g$, i.e. at a metric $g_{a b}$ satisfying $\mathcal{H}_{a b}[g]=0$. The resulting object, which we call $H(h)$, i.e.

$$
H_{a b}(h)=\left.\frac{d}{d \lambda}\right|_{\lambda=0} \mathcal{H}_{a b}[g+\lambda h],
$$

is a third-order linear partial differential operator acting on symmetric tensors $h_{a b}$. By virtue of $H$ being the Hessian of $S$ at a critical point, it is formally self-adjoint. Equivalently we can use the tensor

$$
\mathcal{B}_{a b c}:=\varepsilon_{a b}^{c} \mathcal{H}_{c d}=2 D_{[a} L_{b] c},
$$

where $L_{a b}:=\mathcal{R}_{a b}-\frac{1}{4} g_{a b} \mathcal{R}, \mathcal{R}=g^{a b} \mathcal{R}_{a b}$, and $B_{a b c}$, the linearization of $\mathcal{B}_{a b c}$ at $g$. In the following we shall apply the operators $H_{a b}$ and $B_{a b c}$ only to tensors which are trace-free. With this assumption, $B_{a b c}$ is explicitly given by

$$
B_{a b c}=2\left(D_{[a} \sigma_{b] c}-C_{c[a}^{d} L_{b] d}\right),
$$

where

$$
\sigma_{a b}=D_{(a} D^{c} h_{b) c}-\frac{1}{2} \Delta h_{a b}-\frac{1}{4} g_{a b} D^{c} D^{d} h_{c d}+3 \mathcal{R}_{(a}{ }^{c} h_{b) c}-\frac{3}{4} g_{a b} h^{c d} \mathcal{R}_{c d}-\frac{3}{4} \mathcal{R} h_{a b}
$$

with $\Delta:=g^{a b} D_{a} D_{b}$ the rough Laplacian and

$$
C_{a b}^{d}=\frac{1}{2} g^{c d}\left(D_{a} h_{b c}+D_{b} h_{a c}-D_{c} h_{a b}\right)
$$

We note the following property of TT-tensors:

$$
\Delta h \sim-\frac{1}{4}\left(\operatorname{rot}_{2}\right)^{2} h \quad \text { and } \quad H h \sim \frac{1}{8}\left(\operatorname{rot}_{2}\right)^{3} h, \quad \text { provided } \delta h=0
$$

where " $"$ denotes "modulo curvature terms". Now consider the following sequence

$$
0 \rightarrow \Lambda^{1}(M) \stackrel{L}{\rightarrow} S_{0}^{2}(M, g) \stackrel{H}{\rightarrow} S_{0}^{2}(M, g) \stackrel{\delta}{\rightarrow} \Lambda^{1}(M) \rightarrow 0 .
$$

Proposition [Gasqui \& Goldschmidt]. The sequence (2.10) is an elliptic complex.

Pr o of. Since $g$ satisfies $\mathcal{H}_{a b}[g]=0$, it follows immediately from property (ii) of $\mathcal{H}_{a b}$ that $\delta \circ H=0$. But $L=-\delta^{*}$, and thus

$$
H \circ L=-H \circ \delta^{*}=-H^{*} \circ \delta^{*}=-(\delta \circ H)^{*}=0,
$$

so (2.10) is a complex. Ellipticity at the far left and right of this complex is equivalent to $\delta$ being an underdetermined elliptic operator, which we have checked already. Ellipticity at the second and third place is seen as follows: Denote the symbol of any operator $\mathcal{O}$ by $\overline{\mathcal{O}}(k)$. Then, for example, we want to solve

$$
\bar{H}(k) h=t, \quad \bar{\delta}(k) t=0, \quad k \neq 0 .
$$


Using (2.9) we easily see that $\operatorname{ker} \bar{H}(k) \cap \operatorname{ker} \bar{\delta}(k)=\{0\}$. Thus $S_{0}^{2}\left(\mathbf{R}^{3}, g_{x}\right)=\operatorname{im} \bar{H}(k) \oplus$ $\operatorname{ker} \bar{\delta}(k)=\operatorname{ker} \bar{H}(k) \oplus \operatorname{im} \bar{L}(k)$. The last two relations imply that both (2.11) and

$$
\bar{L}(k) W=h, \quad \bar{H}(k) h=0, \quad k \neq 0
$$

have solutions. This ends the proof of the Proposition.

Next observe that all operations in (2.10) are natural under conformal rescalings of $g$. Thus, when $g^{\prime}=\omega^{2} g$,

$$
\begin{aligned}
L^{\prime} W^{\prime} & =\omega^{2} L W, & W^{\prime} & =\omega^{2} W \\
H^{\prime} h^{\prime} & =\omega^{-1} H h, & h^{\prime} & =\omega^{2} h \\
\delta^{\prime} t^{\prime} & =\omega^{-3} \delta t, & t^{\prime} & =\omega^{-1} t .
\end{aligned}
$$

Therefore the cohomology groups associated with (2.10) only depend on $[g]$, the conformal structure of $g$ which is, of course, locally trivial. Gasqui and Goldschmidt have shown that, if the above complex is interpreted in the sense of local formal power series expansions the cohomologies associated with it, except for the first one, are all trivial.

The first cohomology group of (2.10), namely ker $L$, is the space of (globally defined) conformal Killing vectors on $(M, g)$. The second cohomology group, ker $H / L\left(\Lambda^{1}(M)\right)$, is nothing but the premoduli space of conformally flat structures around $[g]$. The remaining cohomologies do not have an immediate geometrical interpretation. In the compact case, however, we have the following duality.

TheOrem. Let $M$ be compact. Then all cohomologies are finite-dimensional and

$$
\operatorname{ker} L \cong \Lambda^{1}(M) / \delta\left(S_{0}^{2}(M, g)\right), \quad \operatorname{ker} \delta / H\left(S_{0}^{2}(M, g)\right) \cong \operatorname{ker} H / L\left(\Lambda^{1}(M)\right) .
$$

Proof. For the first isomorphism just recall that $\Lambda^{1}(M)=\operatorname{ker} L \oplus \delta\left(S_{0}^{2}(M, g)\right)$. The finite dimensionality of the first summand follows by differential geometry or by noticing that $\operatorname{ker} L=\operatorname{ker} \delta L$ and $\delta L$ is an elliptic operator. For the second isomorphism, define the "generalized Laplacian" $\mathbf{D}$ by $\mathbf{D}=H^{2}+(L \delta)^{3}$. This is a sixth-order, elliptic, selfadjoint operator $\mathbf{D}: S_{0}^{2}(M, g) \rightarrow S_{0}^{2}(M, g)$. Its kernel is finite-dimensional and given by $\operatorname{ker} \mathbf{D}=\operatorname{ker} H \cap \operatorname{ker} \delta$. By an argument analogous to that involving the Hodge Laplacian in Sect. 1, one sees that ker $\mathbf{D}$ is equal to both $\operatorname{ker} \delta / H\left(S_{0}^{2}(M, g)\right)$ and $\operatorname{ker} H / L\left(\Lambda^{1}(M)\right)$. This ends the proof of the Theorem.

3. The main theorem. Gasqui and Goldschmidt [10] have proved the following

Theorem. Let $M$ be simply connected. Then $\operatorname{ker} H / L\left(\Lambda^{1}(M)\right)=\{0\}$.

Our main result is the following

THEOREM. Let $M$ be simply connected and of vanishing second de Rham cohomology. Then $\operatorname{ker} \delta / H\left(S_{0}^{2}(M, g)\right)=\{0\}$. In other words, under the above hypotheses, the expression $t=H(h)$ furnishes the general solution to the equation $\delta t=0$.

Proof. The following method of proof is inspired by the proof of a Lemma in [17, footnote 13], due to Ashtekar, which states that a tracefree Codazzi tensor, i.e. $t_{a b}$ satisfying $\operatorname{rot}_{2} t=0$ on a simply connected space of constant curvature is of the form $t_{a b}=D_{a} D_{b} \alpha-\frac{1}{3} g_{a b} \Delta \alpha$, see also Ferus [8]. Let $\lambda^{a}$ be a conformal Killing vector of $(M, g)$. 
Then, when $\delta t=0$, the 2-form defined by $\varepsilon_{a b}{ }^{d} t_{d c} \lambda^{c}$ is curl-free. Thus

$$
\varepsilon_{a b}{ }^{d} t_{d c} \lambda^{c}=D_{[a} G_{b]} .
$$

Since the space of $\lambda$ 's is finite-dimensional there is a way to linearly assign to each $\lambda$ a covector $G_{b}$. Pick any such assignment. Thus we can write $G_{a}=G_{a}(\lambda)$. By the conformal flatness of $g$, there is, locally, the maximum number of $\lambda$ 's, that is to say 10. Furthermore, since $M$ is simply connected (see e.g. [11]) these $\lambda$ 's can be extended to global conformal Killing vectors. These global conformal Killing vectors can be uniquely characterized by their conformal "Killing data", i.e. the values of $\lambda^{a}, K^{a b}=D^{[a} \lambda^{b]}, D \lambda:=D^{a} \lambda_{a}$ and $D_{b} D^{a} \lambda_{a}$ at any point of $M$. Thus there are tensor fields $U_{a b}, U_{a b c}=U_{a[b c]}, V_{a}, V_{a b}$ such that

$$
G_{b}(\lambda)=U_{b c} \lambda^{c}+U_{b c d} K^{c d}+V_{b}(D \lambda)+V_{b c} D^{c}(D \lambda) .
$$

We now insert this into (3.1) and use the conformal Killing equation satisfied by $\lambda^{a}$, i.e.

$$
D_{a} \lambda_{b}=K_{a b}+\frac{1}{3} g_{a b}(D \lambda)
$$

and some of its corollaries. Since from now on all calculations are purely local, it is possible to choose a conformal gauge for $g_{a b}$ so that the curvature is zero. With this in mind, there holds

$$
\begin{gathered}
D_{a} K_{b c}=-\frac{2}{3} g_{a[b} D_{c]}(D \lambda) \\
D_{a} D_{b}(D \lambda)=0 .
\end{gathered}
$$

Substituting $(3.3,4,5)$ into $(3.1,2)$ and using that the conformal Killing data are arbitrary, we obtain

$$
\begin{aligned}
\varepsilon_{a b}{ }^{d} t_{d c} & =D_{[a} U_{b] c} \\
0 & =D_{[a} U_{b]}{ }^{c d}+U_{[a}^{\left[{ }^{c} \delta_{b]} d\right]} \\
0 & =-\frac{1}{3} U_{[a b]}+D_{[a} V_{b]} \\
0 & =\frac{2}{3} U_{[a b]}^{c}-V_{[a} \delta_{b]}^{c}+D_{[a} V_{b]}^{c} .
\end{aligned}
$$

We solve the equations "from bottom to top" except for (3.8) which turns out to be implied by the remaining relations. Since $U_{a b c}=U_{a[b c]}$, there is the identity

$$
U_{a b c}=U_{[a b] c}+U_{[c a] b}-U_{[b c] a} .
$$

Substituting from (3.9) into the right-hand side of (3.10), inserting into (3.7) and taking a trace of (3.7) we find after some calculation that

$$
\begin{aligned}
U_{b c}= & 3 D_{b} V_{c}+\frac{3}{2} g_{b c}\left(\Delta V_{d}{ }^{d}-D^{d} D^{e} V_{d e}\right)- \\
& -3\left(\Delta V_{(b c)}-D^{d} D_{c} V_{(b d)}\right)+3 D_{b} D^{d} V_{(c d)}- \\
& -3 D_{b} D_{c} V_{d}{ }^{d}-3 D_{b} D^{d} V_{[c d]} .
\end{aligned}
$$

Thus the antisymmetric part $V_{[a b]}$ of $V_{a b}$ does not contribute to $D_{[a} U_{b] c}$. Inserting (3.11) into (3.6), we finally obtain $\left(h_{a b}:=\frac{3}{8} V_{(a b)}-\frac{1}{8} g_{a b} V_{c}^{c}\right)$

$$
\varepsilon_{a b}^{d} t_{d c}=-\frac{1}{8} D_{[a}\left(\Delta h_{b] c}-D^{d} D_{|c|} h_{b] d}\right)+\text { trace-terms. }
$$


Thus $V_{c}^{c}$ drops out of (3.12). Furthermore trace-terms, i.e. terms of the form $g_{c[a} \cdot{ }_{b]}$, do not contribute to $t_{a b}$. So taking the dual of (3.12) with respect to the indices $a$ and $b$, we obtain that $t_{a b}$ has exactly the form of $H_{a b}$, as given from $H_{a b}=\frac{1}{2} \varepsilon_{(a}{ }^{c d} B_{|c d| b)}$ and Equ.'s $(2.6,7)$ in the local gauge where $\mathcal{R}_{a b}=0$. Thus we have proved the Theorem.

We remark that in the next section we give examples of conformally flat structures for which $\operatorname{ker} \delta / H\left(S_{0}^{2}(M, g)\right) \neq\{0\}$.

For the remaining cohomology in $(2.10)$ we have no results except for the duality in the compact case.

4. Compact space-forms. Let $M$ be compact and $g_{a b}$ of constant curvature. In 3 dimensions

$$
\mathcal{R}_{a b c d}=\frac{\mathcal{R}}{3} g_{c[a} g_{b] d} \Longleftrightarrow \mathcal{R}_{a b}=\frac{\mathcal{R}}{3} g_{a b}, \quad \mathcal{R}=\text { const. }
$$

Clearly $g_{a b}$ is conformally flat. We want to compute the space of essential infinitesimal conformally flat deformations of $g_{a b}$, i.e. $\operatorname{ker} H / L\left(\Lambda^{1}(M)\right)$. By the Proposition at the end of the previous section, this amounts to determining $\operatorname{ker} H \cap \operatorname{ker} \delta$. Using $(2.6,7,8)$ and (4.1) we find after a straightforward calculation that, when $D^{a} h_{a b}=0$,

$$
B_{a b c}=-D_{[a} \Delta h_{b] c}+\frac{\mathcal{R}}{3} D_{[a} h_{b] c} .
$$

Furthermore, when $D^{a} h_{a b}=0$

$$
D_{[a} \Delta h_{b] c}=\Delta D_{[a} h_{b] c} .
$$

Thus ker $H / L\left(\Lambda^{1}(M)\right)$ is the same as the space of solutions of the system

$$
\left(\Delta-\frac{\mathcal{R}}{3}\right) D_{[a} h_{b] c}=0, \quad D^{a} h_{a b}=0 .
$$

Suppose first that $\mathcal{R} \geq 0$. Then, contracting the first equation in (4.4) with $D^{[a} h^{b] c}$ and integrating by part, we find that

$$
D_{d} D_{[a} h_{b] c}=0 .
$$

Contracting (4.5) with $g^{d a}$ this implies

$$
\left(\Delta-\frac{\mathcal{R}}{2}\right) h_{a b}=0 .
$$

Upon contraction with $h^{a b}$ and integration this implies

$$
D_{a} h_{b c}=0
$$

and

$$
h_{a b}=0 \quad \text { when } \mathcal{R}>0 .
$$

Thus, in the elliptic case (i.e. $\mathcal{R}>0$ ), the conformal structure defined by $g_{a b}$ is rigid amongst all conformally flat structures on $M$. In fact, this already follows from Kuiper [13] and the results of [1]. When $\mathcal{R}=0,(M, g)$ has to be a flat torus $\mathbf{T}^{3}$ (see Ch. $\mathrm{V}$, Theorem 4.2 of [12]). Corresponding to each $\mathbf{S}^{1}$-factor of $\mathbf{T}^{3}$ there is a covariantly constant vector. Taking tensor products, symmetrizing and subtracting out the trace, we obtain a 5-parameter set of tensors obeying (4.7). Since solutions to (4.7) are uniquely 
determined by their value at some point, these are all solutions to Equ. (4.7). Thus these deformations stay within metrics on $\mathbf{T}^{3}$ conformal to a standard flat one.

Finally we consider the hyperbolic case, $\mathcal{R}<0$. We use the identity, valid when $D^{a} t_{a b}=0$

Thus (4.4) implies

$$
\Delta t_{a b}=-\frac{1}{4}\left(\operatorname{rot}_{2}\right)^{2} t_{a b}+\frac{\mathcal{R}}{2} t_{a b}
$$

$$
\left[\left(\operatorname{rot}_{2}\right)^{2}-\frac{2 \mathcal{R}}{3}\right] \operatorname{rot}_{2} t_{a b}=0
$$

Contracting (4.10) with $\operatorname{rot}_{2} t^{a b}$ and integrating, noting that $\operatorname{rot}_{2}$ is self-adjoint and using $\mathcal{R}<0$, yields

$$
\left(\operatorname{rot}_{2}\right)^{2} t_{a b}=0
$$

which, upon contracting with $t^{a b}$, results in

$$
\operatorname{rot}_{2} t_{a b}=0 \Longleftrightarrow D_{[a} t_{b] c}=0
$$

Thus, in the hyperbolic case, the infinitesimal deformation space ker $H / L\left(\Lambda^{1}(M)\right)$ is isomorphic to the space of traceless Codazzi tensors. This, as Lafontaine [14] has shown, can be non-trivial for certain space forms. By the Mostow rigidity theorem [15], the deformed conformally flat structures can not again be space forms. In fact such deformations (even finite ones) have been constructed (see refs. in [14]), using methods completely beyond the ones of this paper.

\section{References}

[1] L. Bérard-Bergery, J. P. Bourguignon and J. Lafontaine (1975), Déformations localement triviales des variétés riemanniennes, Differential Geometry, Proc. Sympos. Pure Math., vol. XXVII, Part 1, Amer. Math. Soc., Providence, R.I., 3-32.

[2] A. L. Besse (1987), Einstein Manifolds, Springer, Berlin.

[3] E. Calabi (1971), On compact Riemannian manifolds with constant curvature. I, Differential Geometry, Proc. Sympos. Pure Math., vol. III, Amer. Math. Soc., Providence, R.I., $155-180$.

[4] S.-S. Chern, L. Simons (1974), Characteristic forms and geometric invariants, Ann. Math. 99, 48-69, and

S.-S. Chern (1986), On a conformal invariant of three-dimensional manifolds, Aspects of Mathematics and its Applications, J. A. Barroso (Ed.), Elsevier Science Publishers B.V., $245-252$.

[5] Y. Choquet-Bruhat, J. W. York Jr. (1980), The Cauchy Problem, General Relativity and Gravitation, Vol. 1, A. Held (Ed.), Plenum, N.Y., 99-172.

[6] S. Deser (1967), Covariant decomposition of symmetric tensors and the gravitational Cauchy problem, Ann. Inst. Henri Poincaré, VII, 149-188.

[7] S. Deser, R. Jackiw and S. Templeton (1982), Topologically Massive Gauge Theories, Ann. Phys. 140, 372-411.

[8] D. Ferus (1981), A remark on Codazzi tensors in constant curvature spaces, Global Differential Geometry and Global Analysis, D. Ferus et al. (Eds.) LNM 838, Springer, Berlin, 257. 
[9] A. E. Fischer, J. E. Marsden (1977), The manifold of conformally equivalent metrics, Can. J. Math. XXIX, 193-209.

[10] J. Gasqui, H. Goldschmidt (1984), Déformations Infinitésimales des Structures Conformes Plates, Birkhäuser, Basel.

[11] G. Hall (1989), The global extension of local symmetries in general relativity, Class. Quant. Grav. 6, 157-161.

[12] S. Kobayashi, K. Nomizu (1963), Foundations of Differential Geometry Vol. 1, Interscience, Wiley, London.

[13] N. H. Kuiper (1949), On conformally-flat spaces in the large, Ann. Math. 50, 916-924, and

N. H. Kuiper (1950), On compact conformally Euclidean spaces of dimension > 2, Ann. Math. 52, 478-490.

[14] J. P. Lafontaine (1983), Modules de structures conformes plates et cohomologie de groupes discrets, C.R. Acad. Sc. t. 297, Ser.. I, 655-658.

[15] G. D. Mostow (1973), Strong rigidity of locally symmetric spaces, Ann. of Math. Studies 78, Princeton.

[16] J. Schouten (1921), Über die konforme Abbildung $n$-dimensionaler Mannigfaltigkeiten mit quadratischer Maßbestimmung auf eine Mannigfaltigkeit mit euklidischer Maß bestimmung, Math. Z. 11, 58-88.

[17] P. Sommers (1978), The geometry of the gravitational field at spacelike infinity, J. Math. Phys. 19, 549-554.

[18] D. C. Spencer (1969), Overdetermined systems of linear partial differential equations, Bull. AMS 75, 179-239.

[19] F. Warner (1983), Foundations of Differentiable Manifolds and Lie Groups, Springer, Berlin.

[20] J. W. York Jr. (1973), Conformally invariant orthogonal decomposition of symmetric tensors on Riemannian manifolds and the initial-value problem of general relativity, J. Math. Phys. 14, 456-464, and

J. W. York Jr. (1974), Covariant decompositions of symmetric tensors in the theory of gravitation, Ann. Inst. Henri Poincaré 21, 319-332. 